\title{
Communication Behaviour of Small Farmers of Aligarh District on Improved Wheat Technology
}

\author{
Jaspal Singh $^{1}$, Amod Sharma ${ }^{2 *}$, D.B. Tyagi ${ }^{3}$ and S.P. Singh ${ }^{4}$ \\ ${ }^{1}$ C.C.S.R.B.S. College, Bichpuri, Agra Uttar Pradesh, India \\ ${ }^{2}$ Department of Agriculture Economics, NU SASRD, Medziphema Campus, \\ Dimapur, Nagaland, India \\ ${ }^{3}$ S. M. S. Degree College, Abidgarh, Agra Uttar Pradesh, India \\ ${ }^{4} \mathrm{JNKVV}$, Jabalpur, India \\ *Corresponding author
}

\section{A B S T R A C T}

\begin{tabular}{|l|}
\hline Ke y w o r d s \\
$\begin{array}{l}\text { Communication, Small } \\
\text { farmers, Aligarh district, } \\
\text { Wheat technology }\end{array}$ \\
\hline Article Info \\
\hline $\begin{array}{l}\text { Accepted: } \\
\text { 22 April } 2018 \\
\text { Available Online: } \\
10 \text { May } 2018\end{array}$ \\
\hline \hline
\end{tabular}

In this research article an emphasis has been laid on four basic issue viz. socio-agroeconomic profile of small farmers, utilization of communication sources, relationship between socio-agro-economic profile and communication sources and correlation between communication pattern and adoption behaviour of small farmers. The study covers 300 respondents of Aligarh district. Majority of small farmers has 'medium' socio-economic status. In personal cosmopolite channel majority of farmers get communication by training method demonstration and result demonstration. In case of personal localite channel K.V.K., Ag. Supervisor and progressive farmers and in impersonal cosmopolite channels, Radio, film and printed media are the most utilizing communication sources. Different communication channels influence seed technology, fertilizer technology (nitrogenous fertilizers) and weedicides technology are positive component to wheat technology.

\section{Introduction}

Agriculture is the mainstay of Indian economy both in terms of contribution to GDP and the proportion of population dependent upon it. A high growth rate of agriculture is essential for achieving the objective of food security at macro as well as micro levels and also for alleviating poverty level.

While 21 per cent (at constant price) of the GDP in India is contributed by agriculture sector almost two-third of the country's population is dependent on this sector.
Agriculture exports accounts for about 14 per cent of the country's total exports. During the last 4 decades enormous progress has been achieved in agriculture production in the country. India's agriculture from the mid 60's has made significant strides in food grain production which has increased from a level of 72.35 million tonnes to 204.6 million tonnes during 1999-2006 and thus despite of the ever growing population, the need for import of food grains for domestic use has virtually been eliminated (Directorate of Economics and Statistics, Department of Agriculture and Co-operation, Govt. of India). 
Wheat is such a food grain which gives assurance to have success in battle against hunger and poverty. The population of India will be 1.25 million by $2020 \mathrm{AD}$ and by that time India's annual wheat production should reach 109 million tonnes from the present level of 73.7 million tonnes with an area of 28.17 million hectares. The present national average of 2.62 tonnes per hectare of wheat has to be raised to 3.5 tonnes per hectare, which is possible only by proper adoption of presently available package of wheat technology. In U.P. the total wheat producing area during 2005-06 was 9.16 million-hectare and the production was 24.07 million tonnes with the average yield of 2.63 tonnes per hectare (Agricultural Statistics at a Glance, 2007).

It is generally accepted that communication is the basic step in effecting changes in any system in the field of agriculture. The greater the number of in finding sought, the greater is the adoption by contact among farmers with communication sources. Mass media plays an important role in agricultural communication and information sources. There are many sources of information about agriculture technology such as personal cosmopolite channel, personal localite channel and impersonal cosmopolite channel. Source preference and source utilization may also differ at different stages of innovation and decision process depending upon socioeconomic level and infrastructural background of the small farmers.

The present study focuses on the multidimensional aspect of the development of small farmers. The study also highlights the socio-economic feature of the small farmers with a wide and detailed coverage of quite a new approach in the field of agriculture extension research. The study also takes into account the proper use of communication media as a source of information to the small farmers. The knowledge and attitude of small farmer towards new wheat technology has also been studied in depth with following specific objectives.

To examine the extent and utilization of communication sources along with their pattern and effect on the adoption of wheat production technology.

To study the relationship between communication pattern and socio-economic feature of small farmers.

\section{Materials and Methods}

The study was conducted in Aligarh district of U.P. State. Out of the 17 blocks in Aligarh district three blocks namely Dhanipur, Akerabad and Gangiri were selected purposely as the transfer of technology programme of KVK and farmers training centre under CSAUAT Kanpur are more concentrated in these blocks. 10 villages from the selected blocks and 30 small wheat growing farmers from each village were selected randomly. Further, the selected farmers were stratified according to their socio-economic status according to SES scale developed by Trivedi and Pareek (1963) with slight modifications (Table 1).

The $\chi^{2}$ test, ' $\mathrm{t}$ ' test and Pearsonian correlation co-efficient were applied to establish various correlation among different variables used.

\section{Results and Discussion}

\section{Personal cosmopolite channels}

Training was found to be an important communication channel for the adoption of improved farm practices with rank order I (Table 2). Second and third most important channels were noticed as Method demonstration and Result demonstration, respectively for HYV seed, organic manure, nitrogenous fertilizer, phosphatic fertilizer, 
seed rate, depth of sowing, weedicide, time of sowing, irrigation, seed treatment and use of plant protection giving pooled rank order 1.83 and 3 respectively.

\section{Personal localite channels}

Farmers training center or Krishi Vigyan Kendra was found to be important communication channel for adoption of improved farm practices to the small farmers, which has pooled rank order of 2.33 (Table 3). The second important channel was Agricultural Supervisor, which has pooled rank order of 2.5 and the third being Progressive farmer, which has pooled rank order of 3.25 for respective improved practices i.e. HYV seed, organic matter, nitrogenous fertilizer, phosphatic fertilizer, seed rate, depth of sowing, weedicide, time of sowing, irrigation, seed treatment and use of plant protection measures.

\section{Impersonal cosmopolite channels}

Radio was found to be the most important impersonal cosmopolite communication channel for the adoption of improved farm practices to the small farmers with pooled rank order of 1.17 (Table 4).

Films and Printed materials were the other important channel, which have pooled rank order of 2.5 and 2.67 respectively for improved technology of wheat cultivation.

\section{Relationship between communication pattern and socio-economic features}

The overall socio-economic status was found to be significantly correlated with the use of personal localite and impersonal cosmopolite channels. Taking the socio-economic variables separately, caste, education, family, size, size of holding, farm power, change agent linkage, socio-economic status, socio participation were found to be significantly correlated with the use of personal cosmopolite channels while caste, education, family size, income, size of holding, farm power, change agent linkage, contact with extension agencies, socio-economic status and social participation under personal localite channels and the caste, education, family type, income, size of holding, farm power, change agent linkage contact with extension agencies, socioeconomic status and social participation were found to be significantly correlated with the use of impersonal cosmopolite channels (Table 5).

\section{Correlation coefficient between communication pattern and adoption behaviour}

Seed technology, nitrogenous fertilizer and weedicide were significantly correlated with Personal Cosmopolite Channel, Personal Localite Channel and Impersonal Cosmopolite Channel while irrigation and phosphatic fertilizer were significantly correlated with Impersonal Cosmopolite Channel only (Table 6).

Table.1 Distribution of Small farmers according to Socio-economic status

\begin{tabular}{|c|c|c|c|}
\hline S. No. & Socio-economic status & No. of Respondents & Percentage \\
\hline 1. & High Status & 56 & 18.67 \\
\hline 2. & Medium Status & 94 & 31.33 \\
\hline 3. & Low Status & 150 & 50.00 \\
\hline & Total & 300 & 100.00 \\
\hline
\end{tabular}


Table.2 Use of personal cosmopolite channels by the small farmers regarding adoption of wheat technology

\begin{tabular}{|c|c|c|c|c|c|c|c|c|c|c|c|c|c|c|c|c|c|c|c|c|c|c|c|c|c|c|}
\hline \multirow[t]{2}{*}{$\begin{array}{l}\text { Communication } \\
\text { media }\end{array}$} & \multicolumn{2}{|c|}{ HYV seed } & \multicolumn{2}{|c|}{ Seedrate } & \multicolumn{2}{|c|}{$\begin{array}{l}\text { Time of } \\
\text { sowing }\end{array}$} & \multicolumn{2}{|c|}{$\begin{array}{l}\text { Method of } \\
\text { sowing }\end{array}$} & \multicolumn{2}{|c|}{$\begin{array}{l}\text { Depthof } \\
\text { sowing }\end{array}$} & \multicolumn{2}{|c|}{$\begin{array}{c}\text { Seed } \\
\text { treatment }\end{array}$} & \multicolumn{2}{|c|}{$\begin{array}{l}\text { Nitrogenous } \\
\text { ferti }\end{array}$} & \multicolumn{2}{|c|}{$\begin{array}{l}\text { Phosphatic } \\
\text { feri. }\end{array}$} & \multicolumn{2}{|c|}{ Irrigation } & \multicolumn{2}{|c|}{$\begin{array}{l}\text { Organic } \\
\text { matter }\end{array}$} & \multicolumn{2}{|c|}{$\begin{array}{c}\text { Plant } \\
\text { protection }\end{array}$} & \multicolumn{2}{|c|}{ Weedicide } & \multicolumn{2}{|c|}{$\begin{array}{c}\text { Pooledrank } \\
\text { forthe } \\
\text { package }\end{array}$} \\
\hline & No. & $\mathrm{RO}$ & No. & $\mathrm{RO}$ & No. & $\mathrm{RO}$ & No. & $\mathrm{RO}$ & No. & $\mathrm{RO}$ & No. & $\mathrm{RO}$ & No. & $\mathrm{RO}$ & No. & $\mathrm{RO}$ & No. & $\mathrm{RO}$ & No. & $\mathrm{RO}$ & No. & $\mathrm{RO}$ & No. & $\mathrm{RO}$ & No. & $\mathrm{RO}$ \\
\hline $\begin{array}{l}\text { Farmand home } \\
\text { visit }\end{array}$ & $\begin{array}{c}38 \\
(12.67)\end{array}$ & 7 & $\begin{array}{c}22 \\
(7.33)\end{array}$ & 6 & $\begin{array}{c}24 \\
(8.00)\end{array}$ & 7 & $\begin{array}{c}17 \\
(5.67)\end{array}$ & 8 & $\begin{array}{c}7 \\
(2.33)\end{array}$ & 10 & $\begin{array}{c}6 \\
(2.00)\end{array}$ & 9 & $\begin{array}{c}29 \\
(9.67)\end{array}$ & 8 & $\begin{array}{c}21 \\
(7.00)\end{array}$ & 9 & $\begin{array}{c}18 \\
(6.00)\end{array}$ & 9 & $\begin{array}{c}9 \\
(3.00)\end{array}$ & 8 & $\begin{array}{c}7 \\
(2.33)\end{array}$ & 9 & $\begin{array}{c}9 \\
(2.33)\end{array}$ & 7 & 8.08 & 9 \\
\hline $\begin{array}{l}\text { Result } \\
\text { demonstration }\end{array}$ & $\begin{array}{c}122 \\
(40.67)\end{array}$ & 3 & $\begin{array}{c}87 \\
(29.00)\end{array}$ & 3 & $\begin{array}{c}72 \\
(24.00)\end{array}$ & 3 & $\begin{array}{c}64 \\
(21.33)\end{array}$ & 3 & $\begin{array}{c}55 \\
(18.33)\end{array}$ & 3 & $\begin{array}{c}22 \\
(7.33)\end{array}$ & 4 & $\begin{array}{c}102 \\
(34.00)\end{array}$ & 3 & $\begin{array}{c}93 \\
(31.00)\end{array}$ & 3 & $\begin{array}{c}97 \\
(32.33)\end{array}$ & 1 & $\begin{array}{c}72 \\
(24.00)\end{array}$ & 3 & $\begin{array}{c}24 \\
(8.00)\end{array}$ & 4 & $\begin{array}{c}71 \\
(23.67)\end{array}$ & 3 & 3.00 & 3 \\
\hline $\begin{array}{l}\text { Method } \\
\text { demonstration }\end{array}$ & $\begin{array}{c}163 \\
(54.33)\end{array}$ & 2 & $\begin{array}{c}128 \\
(42.67)\end{array}$ & 2 & $\begin{array}{c}109 \\
(36.33)\end{array}$ & 2 & $\begin{array}{c}110 \\
(36.67)\end{array}$ & 2 & $\begin{array}{c}121 \\
(40.33)\end{array}$ & 2 & $\begin{array}{c}36 \\
(12.00)\end{array}$ & 2 & $\begin{array}{c}130 \\
(43.33)\end{array}$ & 2 & $\begin{array}{c}131 \\
(43.67)\end{array}$ & 1 & $\begin{array}{c}61 \\
(20.33)\end{array}$ & 3 & $\begin{array}{c}133 \\
(44.33)\end{array}$ & 2 & $\begin{array}{c}32 \\
(10.67)\end{array}$ & 2 & $\begin{array}{c}110 \\
(36.67)\end{array}$ & 1 & 1.83 & 2 \\
\hline Office calls & $\begin{array}{c}10 \\
(3.33)\end{array}$ & 10 & $\begin{array}{c}0 \\
(0.00)\end{array}$ & 10 & $\begin{array}{c}0 \\
(0.00)\end{array}$ & 10 & $\begin{array}{c}0 \\
(0.00)\end{array}$ & 10 & $\begin{array}{c}0 \\
(0.00)\end{array}$ & 11 & $\begin{array}{c}5 \\
(1.67)\end{array}$ & 10 & $\begin{array}{c}0 \\
(0.00)\end{array}$ & 11 & $\begin{array}{c}0 \\
(0.00)\end{array}$ & 11 & $\begin{array}{c}0 \\
(0.00)\end{array}$ & 10 & $\begin{array}{c}0 \\
(0.00)\end{array}$ & 11 & $\begin{array}{c}7 \\
(2.33)\end{array}$ & 9 & $\begin{array}{c}3 \\
(1.00)\end{array}$ & 8 & 10.08 & 11 \\
\hline Tour/Field trif & $\begin{array}{c}24 \\
(8.00)\end{array}$ & 8 & $\begin{array}{c}19 \\
(6.33)\end{array}$ & 8 & $\begin{array}{c}24 \\
(8.00)\end{array}$ & 7 & $\begin{array}{c}19 \\
(6.33)\end{array}$ & 6 & $\begin{array}{c}12 \\
(4.00)\end{array}$ & 7 & $\begin{array}{c}11 \\
(3.67)\end{array}$ & 8 & $\begin{array}{c}21 \\
(7.00)\end{array}$ & 9 & $\begin{array}{c}23 \\
(7.67)\end{array}$ & 8 & $\begin{array}{c}26 \\
(8.67)\end{array}$ & 7 & $\begin{array}{c}23 \\
(7.67)\end{array}$ & 6 & $\begin{array}{c}13 \\
(4.33)\end{array}$ & 6 & $\begin{array}{c}9 \\
(3.00)\end{array}$ & 7 & 7.25 & 8 \\
\hline Group meeting & $\begin{array}{c}47 \\
(154.67)\end{array}$ & 7 & $\begin{array}{c}21 \\
(7.00)\end{array}$ & 7 & $\begin{array}{c}22 \\
(7.33)\end{array}$ & 8 & $\begin{array}{c}18 \\
(6.00)\end{array}$ & 7 & $\begin{array}{c}11 \\
(3.67)\end{array}$ & 8 & $\begin{array}{c}14 \\
(4.67)\end{array}$ & 6 & $\begin{array}{c}42 \\
(14.00)\end{array}$ & 7 & $\begin{array}{c}33 \\
(11.00)\end{array}$ & 7 & $\begin{array}{c}31 \\
(10.33)\end{array}$ & 5 & $\begin{array}{c}35 \\
(11.67)\end{array}$ & 5 & $\begin{array}{c}9 \\
(3.00)\end{array}$ & 8 & $\begin{array}{c}22 \\
(7.33)\end{array}$ & 5 & 6.58 & 7 \\
\hline Campaign & $\begin{array}{c}11 \\
(3.67)\end{array}$ & 9 & $\begin{array}{c}9 \\
(3.00)\end{array}$ & 9 & $\begin{array}{c}7 \\
(2.33)\end{array}$ & 9 & $\begin{array}{c}8 \\
(2.67)\end{array}$ & 9 & $\begin{array}{c}10 \\
(3.33)\end{array}$ & 9 & $\begin{array}{c}12 \\
(4.00)\end{array}$ & 7 & $\begin{array}{c}11 \\
(3.67)\end{array}$ & 10 & $\begin{array}{c}7 \\
(2.33)\end{array}$ & 10 & $\begin{array}{c}0 \\
(0.00)\end{array}$ & 10 & $\begin{array}{c}3 \\
(1.00)\end{array}$ & 10 & $\begin{array}{c}18 \\
(6.00)\end{array}$ & 5 & $\begin{array}{c}3 \\
(1.00)\end{array}$ & 8 & 8.75 & 10 \\
\hline Training & $\begin{array}{c}152 \\
(50.67)\end{array}$ & 1 & $\begin{array}{c}113 \\
(44.33)\end{array}$ & 1 & $\begin{array}{c}122 \\
(40.67)\end{array}$ & 1 & $\begin{array}{c}127 \\
(42.33)\end{array}$ & 1 & $\begin{array}{c}142 \\
(47.33)\end{array}$ & 1 & $\begin{array}{c}40 \\
(13.33)\end{array}$ & 1 & $\begin{array}{c}140 \\
(46.67)\end{array}$ & 1 & $\begin{array}{c}131 \\
(43.67)\end{array}$ & 1 & $\begin{array}{c}71 \\
(23.67)\end{array}$ & 2 & $\begin{array}{c}141 \\
(47.00)\end{array}$ & 1 & $\begin{array}{c}33 \\
(11.00)\end{array}$ & 1 & $\begin{array}{c}98 \\
(32.67)\end{array}$ & 2 & 1.25 & 1 \\
\hline Exhibition & $\begin{array}{c}67 \\
(22.33)\end{array}$ & 4 & $\begin{array}{c}62 \\
(20.67)\end{array}$ & 4 & $\begin{array}{c}46 \\
(15.33)\end{array}$ & 5 & $\begin{array}{c}17 \\
(5.67)\end{array}$ & 8 & $\begin{array}{c}13 \\
(4.33)\end{array}$ & 6 & $\begin{array}{c}12 \\
(4.00)\end{array}$ & 7 & $\begin{array}{c}50 \\
(16.67)\end{array}$ & 5 & $\begin{array}{c}62 \\
(20.67)\end{array}$ & 5 & $\begin{array}{c}22 \\
(7.33)\end{array}$ & 8 & $\begin{array}{c}8 \\
(2.67)\end{array}$ & 9 & $\begin{array}{c}12 \\
(4.00)\end{array}$ & 7 & $\begin{array}{c}0 \\
(0.00)\end{array}$ & 9 & 6.42 & 6 \\
\hline Fieldday & $\begin{array}{c}55 \\
(18.33)\end{array}$ & 5 & $\begin{array}{c}43 \\
(14.33)\end{array}$ & 5 & $\begin{array}{c}49 \\
(16.33)\end{array}$ & 4 & $\begin{array}{c}32 \\
(10.67)\end{array}$ & 4 & $\begin{array}{c}30 \\
(10.00)\end{array}$ & 4 & $\begin{array}{c}26 \\
(8.67)\end{array}$ & 3 & $\begin{array}{c}44 \\
(14.67)\end{array}$ & 6 & $\begin{array}{c}39 \\
(13.00)\end{array}$ & 6 & $\begin{array}{c}41 \\
(13.67)\end{array}$ & 4 & $\begin{array}{c}42 \\
(14.00)\end{array}$ & 4 & $\begin{array}{c}27 \\
(9.00)\end{array}$ & 3 & $\begin{array}{c}57 \\
(19.00)\end{array}$ & 4 & 4.33 & 4 \\
\hline Kisan Mela & $\begin{array}{c}47 \\
(15.67)\end{array}$ & 6 & $\begin{array}{c}22 \\
(7.33)\end{array}$ & 6 & $\begin{array}{c}38 \\
(12.67)\end{array}$ & 6 & $\begin{array}{c}21 \\
(7.00)\end{array}$ & 5 & $\begin{array}{c}21 \\
(7.00)\end{array}$ & 5 & $\begin{array}{c}18 \\
(6.00)\end{array}$ & 5 & $\begin{array}{c}72 \\
(24.00)\end{array}$ & 4 & $\begin{array}{c}70 \\
(23.33)\end{array}$ & 4 & $\begin{array}{c}27 \\
(9.00)\end{array}$ & 6 & $\begin{array}{c}22 \\
(7.33)\end{array}$ & 7 & $\begin{array}{c}13 \\
(4.33)\end{array}$ & 6 & $\begin{array}{c}12 \\
(4.00)\end{array}$ & 6 & 5.50 & 5 \\
\hline
\end{tabular}

1. Figures in parenthesis indicate percentage of the respondents.

2. Total of percentage in any column when added will exceed 100 since more than one channel was cited by the respondents.

3. $\mathrm{RO}=$ Rank Order 
Table.3 Use of personal localite channels by the small farmers for different improved practices

\begin{tabular}{|c|c|c|c|c|c|c|c|c|c|c|c|c|c|c|c|c|c|c|c|c|c|c|c|c|c|c|}
\hline \multirow[t]{2}{*}{$\begin{array}{c}\text { Communication } \\
\text { media }\end{array}$} & \multicolumn{2}{|c|}{ HYV seed } & \multicolumn{2}{|c|}{ Seed rate } & \multicolumn{2}{|c|}{$\begin{array}{l}\text { Time of } \\
\text { sowing }\end{array}$} & \multicolumn{2}{|c|}{$\begin{array}{l}\text { Method of } \\
\text { sowing }\end{array}$} & \multicolumn{2}{|c|}{$\begin{array}{l}\text { Depthof } \\
\text { sowing }\end{array}$} & \multicolumn{2}{|c|}{$\begin{array}{c}\text { Seed } \\
\text { treatment }\end{array}$} & \multicolumn{2}{|c|}{$\begin{array}{l}\text { Nitrogenous } \\
\text { ferti }\end{array}$} & \multicolumn{2}{|c|}{$\begin{array}{l}\text { Phosphatic } \\
\text { feri. }\end{array}$} & \multicolumn{2}{|c|}{ Imigation } & \multicolumn{2}{|c|}{$\begin{array}{l}\text { Organic } \\
\text { matter }\end{array}$} & \multicolumn{2}{|c|}{$\begin{array}{c}\text { Plant } \\
\text { protection }\end{array}$} & \multicolumn{2}{|c|}{ Weedicide } & \multicolumn{2}{|c|}{$\begin{array}{c}\text { Pooledrank } \\
\text { forthe } \\
\text { package }\end{array}$} \\
\hline & No. & $\mathrm{RO}$ & No. & $\mathrm{RO}$ & No. & $\mathrm{RO}$ & No. & $\mathrm{RO}$ & No. & $\mathrm{RO}$ & No. & $\mathrm{RO}$ & No. & $\mathrm{RO}$ & No. & $\mathrm{RO}$ & No. & $\mathrm{RO}$ & No. & $\mathrm{RO}$ & No. & $\mathrm{RO}$ & No. & $\mathrm{RO}$ & No. & $\mathrm{RO}$ \\
\hline $\begin{array}{l}\text { Agriculture } \\
\text { supervision }\end{array}$ & $\begin{array}{c}72 \\
(24.00)\end{array}$ & 5 & $\begin{array}{c}127 \\
(42.33)\end{array}$ & 1 & $\begin{array}{c}99 \\
(33.00)\end{array}$ & 3 & $\begin{array}{c}117 \\
(39.00)\end{array}$ & 1 & $\begin{array}{c}110 \\
(36.67)\end{array}$ & 2 & $\begin{array}{c}31 \\
(10.33)\end{array}$ & 3 & $\begin{array}{c}121 \\
(40.33)\end{array}$ & 1 & $\begin{array}{c}94 \\
(31.33)\end{array}$ & 1 & $\begin{array}{c}40 \\
(13.33)\end{array}$ & 3 & $\begin{array}{c}36 \\
(12.00)\end{array}$ & 5 & $\begin{array}{c}18 \\
(6.00)\end{array}$ & 3 & $\begin{array}{c}41 \\
(13.67)\end{array}$ & 2 & 2.50 & 2 \\
\hline A.E.O. & $\begin{array}{c}31 \\
(10.33)\end{array}$ & 10 & $\begin{array}{c}29 \\
(9.67)\end{array}$ & 8 & $\begin{array}{c}24 \\
(8.00)\end{array}$ & 9 & $\begin{array}{c}26 \\
(8.67)\end{array}$ & 7 & $\begin{array}{c}19 \\
(6.33)\end{array}$ & 8 & $\begin{array}{c}14 \\
(4.67)\end{array}$ & 9 & $\begin{array}{c}10 \\
(3.33)\end{array}$ & 11 & $\begin{array}{c}16 \\
(5.33)\end{array}$ & 12 & $\begin{array}{c}7 \\
(2.33)\end{array}$ & 10 & $\begin{array}{c}19 \\
(6.33)\end{array}$ & 8 & $\begin{array}{c}11 \\
(3.67)\end{array}$ & 5 & $\begin{array}{c}19 \\
(6.33)\end{array}$ & 8 & 8.75 & 9 \\
\hline B.D.O. & $\begin{array}{c}19 \\
(6.33)\end{array}$ & 12 & $\begin{array}{c}7 \\
(2.33)\end{array}$ & 12 & $\begin{array}{c}11 \\
(3.67)\end{array}$ & 12 & $\begin{array}{c}9 \\
(3.00)\end{array}$ & 11 & $\begin{array}{c}7 \\
(2.33)\end{array}$ & 12 & $\begin{array}{c}11 \\
(3.67)\end{array}$ & 10 & $\begin{array}{c}11 \\
(3.67)\end{array}$ & 10 & $\begin{array}{c}12 \\
(2.33)\end{array}$ & 13 & $\begin{array}{c}5 \\
(1.67)\end{array}$ & 11 & $\begin{array}{c}17 \\
(5.67)\end{array}$ & 9 & $\begin{array}{c}5 \\
(1.66)\end{array}$ & 9 & $\begin{array}{c}9 \\
(3.00)\end{array}$ & 9 & 10.83 & 13 \\
\hline S.M.S & $\begin{array}{c}53 \\
(17.67)\end{array}$ & 7 & $\begin{array}{c}41 \\
(13.67)\end{array}$ & 6 & $\begin{array}{c}47 \\
(15.67)\end{array}$ & 6 & $\begin{array}{c}42 \\
(14.00)\end{array}$ & 5 & $\begin{array}{c}49 \\
(16.33)\end{array}$ & 4 & $\begin{array}{c}35 \\
(11.67)\end{array}$ & 2 & $\begin{array}{c}40 \\
(13.33)\end{array}$ & 6 & $\begin{array}{c}29 \\
(9.67)\end{array}$ & 7 & $\begin{array}{c}44 \\
(14.67)\end{array}$ & 2 & $\begin{array}{c}22 \\
(7.33)\end{array}$ & 6 & $\begin{array}{c}11 \\
(3.67)\end{array}$ & 5 & $\begin{array}{c}21 \\
(7.00)\end{array}$ & 7 & 5.25 & 5 \\
\hline K.V.K. orF.T.C & $\begin{array}{c}121 \\
(40.33)\end{array}$ & 1 & $\begin{array}{c}105 \\
(35.00)\end{array}$ & 4 & $\begin{array}{c}107 \\
(35.67)\end{array}$ & 2 & $\begin{array}{c}109 \\
(36.33)\end{array}$ & 3 & $\begin{array}{c}111 \\
(37.00)\end{array}$ & 1 & $\begin{array}{c}55 \\
(18.33)\end{array}$ & 1 & $\begin{array}{c}121 \\
(40.33)\end{array}$ & 1 & $\begin{array}{c}24 \\
(8.00)\end{array}$ & 10 & $\begin{array}{c}80 \\
(26.67)\end{array}$ & 1 & $\begin{array}{c}120 \\
(40.00)\end{array}$ & 2 & $\begin{array}{c}42 \\
(14.00)\end{array}$ & 1 & $\begin{array}{c}111 \\
(37.00)\end{array}$ & 1 & 2.33 & 1 \\
\hline Co-operatives & $\begin{array}{c}39 \\
(13.00)\end{array}$ & 8 & $\begin{array}{c}21 \\
(7.00)\end{array}$ & 10 & $\begin{array}{c}22 \\
(7.33)\end{array}$ & 10 & $\begin{array}{c}6 \\
(2.00)\end{array}$ & 12 & $\begin{array}{c}27 \\
(9.00)\end{array}$ & 5 & $\begin{array}{c}30 \\
(10.00)\end{array}$ & 4 & $\begin{array}{c}75 \\
(25.00)\end{array}$ & 3 & $\begin{array}{c}34 \\
(11.33)\end{array}$ & 5 & $\begin{array}{c}25 \\
(8.33)\end{array}$ & 5 & $\begin{array}{c}21 \\
(7.00)\end{array}$ & 7 & $\begin{array}{c}7 \\
(2.33)\end{array}$ & 7 & $\begin{array}{c}0 \\
(0.00)\end{array}$ & 11 & 7.25 & 8 \\
\hline $\begin{array}{l}\text { Farmers } \\
\text { organization }\end{array}$ & $\begin{array}{c}24 \\
(8.00)\end{array}$ & 11 & $\begin{array}{c}22 \\
(7.33)\end{array}$ & 9 & $\begin{array}{c}18 \\
(6.00)\end{array}$ & 11 & $\begin{array}{c}23 \\
(7.67)\end{array}$ & 8 & $\begin{array}{c}9 \\
(3.00)\end{array}$ & 11 & $\begin{array}{c}1 \\
(0.33)\end{array}$ & 12 & $\begin{array}{c}40 \\
(13.33)\end{array}$ & 6 & $\begin{array}{c}27 \\
(9.00)\end{array}$ & 8 & $\begin{array}{c}0 \\
(0.00)\end{array}$ & 13 & $\begin{array}{c}19 \\
(6.33)\end{array}$ & 8 & $\begin{array}{c}0 \\
(0.00)\end{array}$ & 10 & $\begin{array}{c}0 \\
(0.00)\end{array}$ & 11 & 9.83 & 10 \\
\hline Panchayats & $\begin{array}{c}13 \\
(4.33)\end{array}$ & 13 & $\begin{array}{c}9 \\
(3.00)\end{array}$ & 11 & $\begin{array}{c}5 \\
(1.67)\end{array}$ & 13 & $\begin{array}{c}5 \\
(1.67)\end{array}$ & 13 & $\begin{array}{c}11 \\
(3.67)\end{array}$ & 10 & $\begin{array}{c}3 \\
(1.00)\end{array}$ & 11 & $\begin{array}{c}25 \\
(8.33)\end{array}$ & 9 & $\begin{array}{c}25 \\
(8.33)\end{array}$ & 9 & $\begin{array}{c}2 \\
(0.66)\end{array}$ & 12 & $\begin{array}{c}22 \\
(7.33)\end{array}$ & 6 & $\begin{array}{c}6 \\
(2.00)\end{array}$ & 8 & $\begin{array}{c}3 \\
(1.00)\end{array}$ & 10 & 10.42 & 12 \\
\hline $\begin{array}{l}\text { Progressive } \\
\text { farmers }\end{array}$ & $\begin{array}{c}109 \\
(36.33)\end{array}$ & 2 & $\begin{array}{c}111 \\
(37.00)\end{array}$ & 2 & $\begin{array}{c}109 \\
(36.33)\end{array}$ & 1 & $\begin{array}{c}41 \\
(13.67)\end{array}$ & 6 & $\begin{array}{c}25 \\
(8.33)\end{array}$ & 6 & $\begin{array}{c}21 \\
(7.00)\end{array}$ & 5 & $\begin{array}{c}66 \\
(22.00)\end{array}$ & 4 & $\begin{array}{c}66 \\
(22.00)\end{array}$ & 3 & $\begin{array}{c}29 \\
(9.67)\end{array}$ & 4 & $\begin{array}{c}127 \\
(42.33)\end{array}$ & 1 & $\begin{array}{c}23 \\
(7.67)\end{array}$ & 2 & $\begin{array}{c}36 \\
(12.00)\end{array}$ & 3 & 3.25 & 3 \\
\hline Friends & $\begin{array}{c}77 \\
(25.67)\end{array}$ & 4 & $\begin{array}{c}34 \\
(11.33)\end{array}$ & 7 & $\begin{array}{c}27 \\
(9.00)\end{array}$ & 8 & $\begin{array}{c}13 \\
(4.33)\end{array}$ & 9 & $\begin{array}{c}19 \\
(6.33)\end{array}$ & 8 & $\begin{array}{c}18 \\
(6.00)\end{array}$ & 7 & $\begin{array}{c}32 \\
(10.67)\end{array}$ & 8 & $\begin{array}{c}32 \\
(10.67)\end{array}$ & 6 & $\begin{array}{c}22 \\
(7.33)\end{array}$ & 6 & $\begin{array}{c}55 \\
(18.33)\end{array}$ & 3 & $\begin{array}{c}17 \\
(5.67)\end{array}$ & 4 & $\begin{array}{c}33 \\
(11.00)\end{array}$ & 4 & 6.16 & 7 \\
\hline Neighbour & $\begin{array}{c}82 \\
(27.33)\end{array}$ & 3 & $\begin{array}{c}109 \\
(36.33)\end{array}$ & 3 & $\begin{array}{c}98 \\
(32.67)\end{array}$ & 4 & $\begin{array}{c}110 \\
(36.67)\end{array}$ & 2 & $\begin{array}{c}65 \\
(21.67)\end{array}$ & 3 & $\begin{array}{c}16 \\
(5.33)\end{array}$ & 8 & $\begin{array}{c}107 \\
(35.67)\end{array}$ & 2 & $\begin{array}{c}90 \\
(30.00)\end{array}$ & 2 & $\begin{array}{c}20 \\
(6.67)\end{array}$ & 8 & $\begin{array}{c}52 \\
(17.33)\end{array}$ & 4 & $\begin{array}{c}11 \\
(3.67)\end{array}$ & 5 & $\begin{array}{c}21 \\
(7.00)\end{array}$ & 7 & 4.25 & 4 \\
\hline Farmleader & $\begin{array}{c}62 \\
(20.67)\end{array}$ & 6 & $\begin{array}{c}61 \\
(20.33)\end{array}$ & 5 & $\begin{array}{c}52 \\
(17.33)\end{array}$ & 5 & $\begin{array}{c}49 \\
(16.33)\end{array}$ & 4 & $\begin{array}{c}17 \\
(5.67)\end{array}$ & 9 & $\begin{array}{c}55 \\
(18.33)\end{array}$ & 1 & $\begin{array}{c}44 \\
(14.67)\end{array}$ & 5 & $\begin{array}{c}40 \\
(13.33)\end{array}$ & 4 & $\begin{array}{c}18 \\
(6.00)\end{array}$ & 9 & $\begin{array}{c}14 \\
(4.67)\end{array}$ & 10 & $\begin{array}{c}23 \\
(7.67)\end{array}$ & 2 & $\begin{array}{c}29 \\
(9.67)\end{array}$ & 5 & 5.42 & 6 \\
\hline Relations & $\begin{array}{c}36 \\
(12.00)\end{array}$ & 9 & $\begin{array}{c}22 \\
(7.33)\end{array}$ & 9 & $\begin{array}{c}29 \\
(9.67)\end{array}$ & 7 & $\begin{array}{c}12 \\
(4.00)\end{array}$ & 10 & $\begin{array}{c}24 \\
(8.00)\end{array}$ & 7 & $\begin{array}{c}19 \\
(6.33)\end{array}$ & 6 & $\begin{array}{c}34 \\
(11.33)\end{array}$ & 7 & $\begin{array}{c}22 \\
(7.33)\end{array}$ & 11 & $\begin{array}{c}21 \\
(7.00)\end{array}$ & 7 & $\begin{array}{c}9 \\
(3.00)\end{array}$ & 11 & $\begin{array}{c}9 \\
(3.00)\end{array}$ & 6 & $\begin{array}{c}23 \\
(7.67)\end{array}$ & 6 & 8.00 & 9 \\
\hline
\end{tabular}

1. Figures in parenthesis indicate percentage of the respondents.

2. Total of percentage in any column when added will exceed 100 since more than one channel was cited by the respondents.

3. $\mathrm{RO}=$ Rank Order 
Table.4 Use of impersonal cosmopolite channels by the small farmers for different improved farm practices package of percentages of wheat

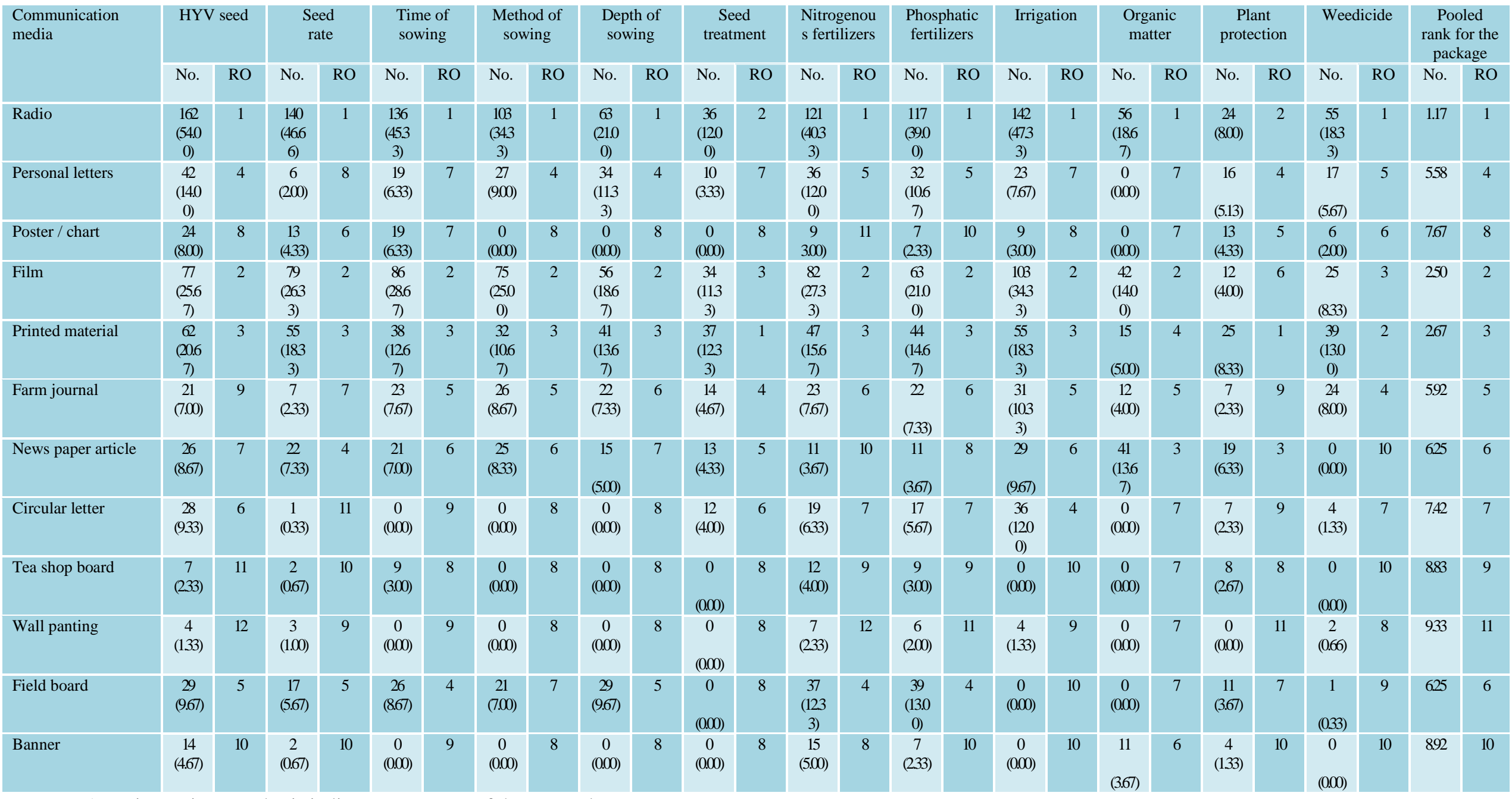

1. Figures in parenthesis indicate percentage of the respondents.

2. Total of percentage in any column when added will exceed 100 since more than one channel was cited by the respondents.

3. $\mathrm{RO}=$ Rank Order 
Table.5 Relationship between communication pattern and socio-economic features

\begin{tabular}{|c|l|c|c|c|}
$\begin{array}{c}\text { S. } \\
\text { No. }\end{array}$ & Socio-economic Characteristic & \multicolumn{3}{|c|}{ Communication Channels } \\
\cline { 2 - 5 } & & PCC & PLC & ICC \\
\hline 1. & Age $(\mathrm{r})$ & $-0.094 \mathrm{NS}$ & $-0.077 \mathrm{NS}$ & $-0.079 \mathrm{NS}$ \\
\hline 2. & Caste $\left(\mathrm{x}^{2}\right)$ & $65.270^{* *}$ & $72.112^{* *}$ & $66.912^{* *}$ \\
\hline 3. & Education $\left(\mathrm{x}^{2}\right)$ & $78.512^{* *}$ & $108.313^{* *}$ & $98.918^{* *}$ \\
\hline 4. & Family type $\left(\mathrm{x}^{2}\right)$ & $8.205 \mathrm{NS}$ & $8.545 \mathrm{NS}$ & $23.550 \mathrm{NS}$ \\
\hline 5. & Family Size $\left(\mathrm{x}^{2}\right)$ & $27.815^{* *}$ & $33.355^{* *}$ & $5.216 \mathrm{NS}$ \\
\hline 6. & Income $(\mathrm{r})$ & $0.072 \mathrm{NS}$ & $0.338^{* *}$ & $0.415^{* *}$ \\
\hline 7. & Size of Holding $(\mathrm{r})$ & $0.162^{* *}$ & $0.241^{* *}$ & $0.203^{* *}$ \\
\hline 8. & Farm Power $(\mathrm{r})$ & $0.228^{* *}$ & $1.168^{* *}$ & $0.399^{* *}$ \\
\hline 9. & Change agent linkage $\left(\mathrm{x}^{2}\right)$ & $92.912^{* *}$ & $122.543^{* *}$ & $116.615^{* *}$ \\
\hline 10. & Contact with extension agencies $\left(\mathrm{x}^{2}\right)$ & $15.882 \mathrm{NS}$ & $110.545^{* *}$ & $92.512^{* *}$ \\
\hline 11. & Socio-economic status $\left(\mathrm{x}^{2}\right)$ & $0.389^{* *}$ & $0.114^{* *}$ & $0.505^{* *}$ \\
\hline 12. & Social participation $(\mathrm{r})$ & $93.554^{*}$ & $142.22^{* *}$ & $125.544^{* *}$ \\
\hline
\end{tabular}

** Significant at $1 \%$ level, NS-Not- significant, * Significant at $5 \%$ level

Table.6 Correlation coefficient between communication pattern and adoption behaviour

\begin{tabular}{|c|l|c|c|c|}
\hline S. & \multicolumn{1}{|c|}{ Adoption behaviour } & \multicolumn{3}{|c|}{ Communication Channels } \\
\cline { 2 - 5 } No. & & PCC & PLC & ICC \\
\hline 1. & Seed technology & $0.332^{* *}$ & $0.402^{* *}$ & $0.325^{* *}$ \\
\hline 2. & Nitrogenous fertilizers & $0.177^{* *}$ & $0.325^{* *}$ & $0.367^{* *}$ \\
\hline 3. & Phosphatic fertilizers & $0.082 \mathrm{NS}$ & $0.024 \mathrm{NS}$ & $0.302^{* *}$ \\
\hline 4. & Pottasic fertilizers & $0.054 \mathrm{NS}$ & $0.012 \mathrm{NS}$ & $0.023 \mathrm{NS}$ \\
\hline 5. & Plant Protection technology & $0.011 \mathrm{NS}$ & $0.266^{* *}$ & $0.304^{* *}$ \\
\hline 6. & Weedicides & $0.198^{* *}$ & $0.186^{* *}$ & $0.170^{* *}$ \\
\hline 7. & Irrigation & $0.056 \mathrm{NS}$ & $0.089 \mathrm{NS}$ & $0.222^{* *}$ \\
\hline
\end{tabular}

** Significant at $1 \%$ level, NS-Not-significant

The significant result clearly indicates that the extent of adoption of agricultural technology is certainly influenced by the use and availability of different communication channels to the farmers. Though, the phosphatic and potassic fertilizers, insecticides and irrigation are the four important technological components affecting wheat production, which in this study are not found significant for all the communication channels.

The present study reveals that training, method demonstration, result demonstration and field day were the most important Personal cosmopolite channels used by the small farmers while on the other hand the least used channels were office calls, campaign, farm and home visit.

It was also observed that the most important Personal localite channels, which have been used by the small farmers, were $\mathrm{KVK}$, agricultural supervisor and progressive farmers, while least used channels were B.D.O., Panchayat and farmers organizations. Impersonal cosmopolite channels i.e. Radio, Film, Printed material and personal letter 
were mostly used by the small farmers while the least used channel were wall painting, banner and tea shop board.

The results of the study highlights that the use and effectiveness of communication channels very much depends on the general background of the small farmers. In other words the variables which were significantly correlated with the communication channels are the indication of the influence over the acceptance and overall adoption of new technology.

Seed technology, fertilizer technology (nitrogenous) and weedicide are the three important components of wheat technology, which had shown positive trend so far the adoption of these practices are concerned. The use and availability of communication channels influence the adoption of these practices positively.

\section{References}

Jha, P.N. and G.S. Skaktawat (1973) "Adoption behaviour of farmers towards Hybrid Bajra cultivation" Indian Journal of Ext. Edu. 8 (1-2) 2429

Singh, S.P., Y.P. Singh and Vikram Sharma (2000) "Effectiveness of different communication media for transfer of wheat production technology. Indian Journal of Adult Education vol. 61 No. 3 July-Sept. 2000.

\section{How to cite this article:}

Jaspal Singh, Amod Sharma, D.B. Tyagi and S.P. Singh. 2018. Communication Behaviour of Small Farmers of Aligarh District on Improved Wheat Technology. Int.J.Curr.Microbiol.App.Sci. 7(05): 3087-3094. doi: https://doi.org/10.20546/ijcmas.2018.705.360 